\title{
ON SOME PROBLEMS IN THE THEORY OF RIEMANN ZETA-FUNCTION
}

\author{
YU.F. KOROBEINIK \\ Dedicated to the memory of professor \\ Igor' Fedorovich Krasichkov-Ternovskii
}

\begin{abstract}
We determine the principal value of some integrals related to Riemann zetafunction. We propose a probably new conjecture implying the famous Riemann's hypothesis on the absence of zeroes of zeta-function in the half-plane $\operatorname{Re} z>1 / 2$, as well as some other facts in the theory of zeta-function.
\end{abstract}

Keywords: Riemann zeta-function, theory of residues.

Mathematics Subject Classification: 14G10

\section{INSTEAD OF INTRODUCTION}

I would like to devote this paper to the memory of my old friend and an outstanding mathematician Igor' Fedorovich Krasichkov-Ternovskii. The fate threw us together somewhen between the end of $50 \mathrm{~s}$ and the beginning of $60 \mathrm{~s}$. At that time, being a young candidate of sciences, I visited punctually the seminars of A.F. Leontiev first in MEI and later in Steklov Institute of Mathematics during my often trips to Moscow. After the end of weekly seminars in Mondays, 11 am, the participants, among those there was his postgraduated student I.F. Krasichkov-Ternovskii, graduated from MSU, crowded to Lenin's Mountains, being lead by A.F. Leontiev, to participate in a scientific seminar on the theory of functions of complex variables headed by professor A.I. Markushevich in the faculty of mechanics and mathematics of MSU.

During those years I visited repeatedly Moscow flats of Igor' Fedorovich, in Matveevskaya street and Universitetsky avenue. Our acquaintance continued in Ufa, where Igor' Fedorovich moved following Alexei Fedorovich after a successful defense of candidate thesis. It should be mentioned that although the first pupils of A.F. Leontiev, Yu.N. Frolov and V.P. Gromov, who defended his doctoral theses with an active help of their scientific supervisor, left Ufa and returned back to Moscow soon after their defenses, Igor' Fedorovich stayed in Ufa also after the defense of a doctoral thesis in Kharkov. He stayed in Ufa till the end of his scientific activity and returned back to Moscow just in the end of his life when he was seriously ill.

During all this time (from 70s till 90s of the last century) I repeatedly met Igor' Fedorovich in various schools, conferences, seminars and other mathematical events in many cities of our immense Motherland. He was the first who communicated me a mournful news on the death of Alexey Fedorovich Leontiev in 1987 and I bid farewell to Alexey Fedorovich already in Moscow in his flat near MEI.

It would be natural to write this paper in my previous scientific field well known to the residents of Ufa (linear equations of infinite order, absolutely representing systems and their applications to partial differential equations), where I repeatedly employed and cited deep

Yu.F. Korobeinik, On some problems in the theory of Riemann's Zeta-Function.

(C) KorobeiniK YU.F. 2015.

Submitted September 12, 2015. 
results of Igor' Fedorovich. However, after long reflections, I decided to provide here the results on the theory of Riemann zeta-function, which I study (hard but not very successful) during last 15 years. I hope, that this paper will attract the attention of Ufa mathematicians to this subject probably fresh for them.

\section{ON MEAN VALUES OF SOME INTEGRALS RELATED WiTH ZETA-FUNCTION}

Let $\varphi \in(0, \pi / 2]$ and $\Gamma_{\varphi}=\left\{z=\frac{1}{2}+\rho e^{i \varphi},-\infty<\rho<+\infty\right\}$ be a line passing through the point $A=1 / 2$ with the slope $\varphi$ to the real axis (more precisely, to its real semi-axis). Let $T \in(0,+\infty)$ and $\Gamma_{\varphi, T}$ be the segment of the line $\Gamma_{\varphi}$ with the beginning at the point $B_{T, \varphi}=\left\{\frac{1}{2}-T e^{i \varphi}\right\}$ and the end at the point $C_{T, \varphi}=\left\{\frac{1}{2}+T e^{i \varphi}\right\}$. Let us consider the integral

$$
\mathcal{I}_{T, \varphi}:=\int_{B_{T, \varphi}}^{C_{T, \varphi}} \frac{\zeta^{\prime}(z)}{\zeta(z)} d z
$$

taken over segment $\Gamma_{\varphi, T}$. We assume first that $\zeta\left(\frac{1}{2}+T e^{i \varphi}\right) \neq 0$; by the known functional equation $\zeta(z)=a(z) \zeta(1-z)$, where $a(z)=z(2 \pi)^{z-1} \sin \frac{\pi z}{2} \Gamma(1-z)$, then $\zeta\left(\frac{1}{2}-T e^{i \varphi}\right) \neq 0$. Let $z_{1}, z_{2}, \ldots, z_{p}$ be the possible zeroes of $\zeta(z)$ in the segment $\left[\frac{1}{2}-T e^{i \varphi}, \frac{1}{2}\right]$. Then by the above mentioned functional equation for $\zeta(z), 1-z_{1}, 1-z_{2}, \ldots, 1-z_{p}$ are the zeroes of $\zeta(z)$ in the segment $\left[\frac{1}{2}, \frac{1}{2}+T e^{i \varphi}\right]$, and $z_{j}$ is the zero of the same order as $1-z_{j}, j=1,2, \ldots, p$.

Let $\Gamma_{\varphi, T}^{(1)}$ be the curve beginning at the point $\frac{1}{2}-T e^{i \varphi}$ and ending at the point $\frac{1}{2}$ formed by the semi-circles $C_{T, \varphi}^{1, r, j}$ of raidus $r>0$ and the following pieces of the segment $\left[\frac{1}{2}-T e^{i \varphi}, \frac{1}{2}\right]$ :

$$
\left[\frac{1}{2}-T e^{i \varphi}, z_{1}-r e^{i \varphi}\right],\left[z_{1}+r e^{i \varphi}, z_{2}-r e^{i \varphi}\right], \ldots\left[z_{p-1}+r e^{i \varphi}, z_{p}-r e^{i \varphi}\right],\left[z_{p}+-r e^{i \varphi}, \frac{1}{2}\right]
$$

At that, we choose $r>0$ small enough so that the semi-circles $C_{T, \varphi}^{1, r, j}$ intersect neither each other no the end-points $\frac{1}{2}-T e^{i \varphi}$ and $\frac{1}{2}$. We choose all the semi-circles $C_{T, \varphi}^{1, r, j}$ so that their centers $z_{j}$ are to the left while passing curve $\Gamma_{\varphi, T}^{(1)}$ from the point $\frac{1}{2}-T e^{i \varphi}$ to $\frac{1}{2}$.

By $\Gamma_{\varphi, T}^{(2)}$ we denote the curve symmetric to $\Gamma_{\varphi, T}^{(1)}$ with respect to the point $\frac{1}{2}$, i.e., the curve beginning at the point $\frac{1}{2}$ and ending at $\frac{1}{2}+T e^{i \varphi}$ formed by the pieces of the segment $\left[\frac{1}{2}, \frac{1}{2}+T e^{i \varphi}\right]$ and semi-circles $C_{T, \varphi}^{2, r, j}, j=1,2, \ldots, p$, of radius $r$ centered at points $1-z_{j}$. At that, the centers are to the right while passing $\Gamma_{\varphi, T}^{(2)}$ from $\frac{1}{2}$ to $\frac{1}{2}+T e^{i \varphi}$. We let

$$
\begin{aligned}
\mathcal{I}_{r}^{T, \varphi} & :=\int_{\Gamma_{\varphi, T}^{(1)}} \frac{\zeta^{\prime}(z)}{\zeta(z)} d z+\int_{\Gamma_{\varphi, T}^{(2)}} \frac{\zeta^{\prime}(z)}{\zeta(z)} d z=\int_{\Gamma_{\varphi, T}^{(1)}} \frac{\zeta^{\prime}(z)}{\zeta(z)} d z+\int_{\Gamma_{\varphi, T}^{(1)}} \frac{\zeta^{\prime}(1-w)}{\zeta(1-w)} d w \\
& =\int_{\Gamma_{\varphi, T}^{(1)}}\left[\frac{\zeta^{\prime}(z)}{\zeta(z)}+\frac{\zeta^{\prime}(1-z)}{\zeta(1-z)}\right] d z .
\end{aligned}
$$

As it is known, the integration variable for a proper or a contour integral can be denoted by any symbol. By the functional Riemann equation we get easily that if $\zeta(z) \zeta(1-z) a(z) \neq 0$, 
then

$$
\frac{\zeta^{\prime}(z)}{\zeta(z)}+\frac{\zeta^{\prime}(1-z)}{\zeta(1-z)}=\frac{a^{\prime}(z)}{a(z)}
$$

It yields $\mathcal{I}_{r}^{T, \varphi}:=\int_{\Gamma_{\varphi, T}^{(1)}} \frac{a^{\prime}(z)}{a(z)} d z$ and therefore,

$$
\lim _{r \rightarrow+0} \mathcal{I}_{r}^{T, \varphi}=\text { v.p. } \int_{\frac{1}{2}-T e^{i \varphi}}^{\frac{1}{2}+T e^{i \varphi}} \frac{\zeta^{\prime}(z)}{\zeta(z)} d z=\int_{\frac{1}{2}-T e^{i \varphi}}^{\frac{1}{2}} \frac{a^{\prime}(z)}{a(z)} d z=-\ln a\left(\frac{1}{2}-T e^{i \varphi}\right)
$$

The functional equation for $\zeta(z)$ yields that $a(1 / 2)=1$. Hence, $\forall \varphi \in(0, \pi / 2], \forall T \in(0,+\infty)$

$$
\text { v.p. } \int_{\frac{1}{2}-T e^{i \varphi}}^{\frac{1}{2}+T e^{i \varphi}} \frac{\zeta^{\prime}(z)}{\zeta(z)} d z=-\ln a\left(\frac{1}{2}-T e^{i \varphi}\right) .
$$

In the case $\zeta\left(\frac{1}{2}-T e^{i \varphi}\right)=0$ (then also $\left.\zeta\left(\frac{1}{2}+T e^{i \varphi}\right)=0\right)$, while constructing curve $\Gamma_{\varphi, T}^{(1)}$ one has to use also a quarter of the circle of radius $r>0$ centered at the point $\frac{1}{2}-T e^{i \varphi}$. A similar change (i.e., construction of a quarter of the circle of radius $r>0$ centered at the point $\left.\frac{1}{2}+T e^{i \varphi}\right)$ is made for curve $\Gamma_{\varphi, T}^{(2)}$, which is symmetric for $\Gamma_{\varphi, T}^{(1)}$ w.r.t. the point $\frac{1}{2}$. Arguing in the same way, here we arrive at formula (1). Thus, we have proved the following theorem.

Theorem 1. If $\varphi \in(0, \pi / 2]$ and $T \in(0,+\infty)$, then formula (1) holds true.

Remark 1. In the exceptional case $\varphi=0$, the situation becomes a little bit more complicated because of the presence of trivial zeroes $-2 \pi k, k=1,2, \ldots$, and a simple pole at $z=1$ of function $\zeta(z)$, as well as because of simple zeroes $2 m, m=0,1, \ldots$, and simple poles $2 k-1$, $k=1,2, \ldots$ of function $a(z)$. However, in this case by similar arguments one get an analogue of formula (1).

Remark 2. Theorem 1 has application in the theory of Riemann zeta-function. More precisely, the mean value of a more general integral

$$
v . p . \int_{\frac{1}{2}-T e^{i \varphi}}^{\frac{1}{2}+T e^{i \varphi}} h(z) \frac{\zeta^{\prime}(z)}{\zeta(z)} d z
$$

is employed, where function $h(z)$ is (locally) analytic in the vicinity of straight line $\Gamma_{\varphi}$ and satisfies the relation $h(z)=h(1-z), \forall z \in \Gamma_{\varphi}$. This mean value is calculated easily for $\varphi \in$ $(0, \pi / 2]$ by the methods presented in this section.

\section{ON A CONJECTURE IN THE THEORY OF ZETA-FUNCTION}

Following [1], we call a number $T$ in $(0,+\infty) \zeta$-regular if $\zeta(\sigma+i T) \neq 0, \forall \sigma \in \mathbb{R}$. We recall some well-known properties of zeta-function $\zeta(z)$ described, for instance, in monographs [1], [2]. As it is known, Riemann zeta function is defined first in the half-plane $G_{1}:=\{z: \operatorname{Re} z>1\}$ as the sum of a usual Dirichlet series $\sum_{n=1}^{\infty} n^{-z}$ or as the inverse to the infinite Euler-Riemann product convergent absolutely in the same half-plane. Function $\zeta(z)$ can be continued analytically to the whole complex plane except the point $z=1$, at which it has a simple pole. Finally,

$$
\zeta(\bar{z})=\overline{\zeta(z)} \text { and } \frac{\zeta^{\prime}(\bar{z})}{\zeta(\bar{z})}=\overline{\left(\frac{\zeta^{\prime}(z)}{\zeta(z)}\right)}
$$

$\forall z \in \mathbb{C} \backslash\{1\}$. We shall employ the latter property in the next section. This identity makes sense if $\zeta(z) \neq 0$. We note that these identities are valid for wider classes of functions; for instance, 
for functions being the unique analytic continuations of either a sum of a power series with a non-zero convergence radius and real Taylor coefficients or of the sum of a Dirichlet series $\sum_{n=1}^{\infty} b_{n} e^{-\lambda_{n} z}$ with the abscissa of absolute convergence $a<+\infty$, real exponents $\lambda_{n}$ and real coefficients $b_{n}, n \geqslant 1$.

Let $\varphi=\pi / 2, h \in(0,+\infty), T \in(0,+\infty)$ be a $\zeta$-regular value. We let $B:=\frac{1}{2}-i T$, $C:=h-i T, D:=h+i T, E:=\frac{1}{2}+i T, \Gamma:=\bigcup_{k=1}^{4} \Gamma_{k}$, where $\Gamma_{1}:=[B, C], \Gamma_{2}:=[C, D]$, $\Gamma_{3}:=[D, E], \Gamma_{4}:=\lim _{r \rightarrow+0} \Gamma_{4}^{r}$, and rectifiable Jordan curve $\Gamma_{4}^{r}$ beginning at point $E$ and ending at point $B$ is constructed at it was described in Section 2 . That is, $\Gamma_{4}^{r}$ is formed by semi-cirles $C_{T, \varphi}^{s, r, j}(s=1,2)$ of a sufficiently small radius and centered at zeroes $z_{j}$ of function $\zeta(z)$ located in $T_{\varphi}$ as well as of some pieces of the segment $[E, B]$. At that, as it was shown in Section 2 ,

$$
\text { v.p. } \int_{\Gamma_{4}} \frac{\zeta^{\prime}(z)}{\zeta(z)} d z=\lim _{r \rightarrow+0} \int_{\Gamma_{4}^{r}} \frac{\zeta^{\prime}(z)}{\zeta(z)} d z=\ln a\left(\frac{1}{2}-i T\right) .
$$

By the theorem on logarithmic residue

$$
2 \pi\left[N_{0}(T)+N_{1}(T)-1\right]=\operatorname{Im} \int_{\Gamma} \frac{\zeta^{\prime}(z)}{\zeta(z)} d z=\sum_{k=1}^{4} \operatorname{Im} \int_{\Gamma_{k}} \frac{\zeta^{\prime}(z)}{\zeta(z)} d z=\sum_{k=1}^{4} \operatorname{Im} I_{k} .
$$

Here, as in [1, the symbol $N_{0}(T)$ stands for the number of zeroes of function $\zeta(z)$ (counting their multiplicities) in the interval $\left(\frac{1}{2}, \frac{1}{2}+i T,\right)$, and $N_{1}(T)$ is the number of all (possible) zeroes of functions $\zeta(z)$ (also counting the multiplicities) lying inside the rectangle with vertices $B$, $C, D, E$. At that,

$$
\operatorname{Im} I_{1}=-\operatorname{Im} \int_{C}^{B} \frac{\zeta^{\prime}(z)}{\zeta(z)} d z=-\operatorname{Im} \int_{h}^{1 / 2} \frac{\zeta^{\prime}(x-i T)}{\zeta(x-i T)} d x
$$

that implies $\operatorname{Im} I_{1}+\operatorname{Im} I_{3}=2 \operatorname{Im} I_{3}$. Then,

$$
\operatorname{Im} \int_{C}^{D} \frac{\zeta^{\prime}(z)}{\zeta(z)} d z=\operatorname{Im}\left\{\int_{-T}^{0} \frac{\zeta^{\prime}(h+i \tau)}{\zeta(h+i \tau)} i d \tau+\int_{0}^{T} \frac{\zeta^{\prime}(h+i \tau)}{\zeta(h+i \tau)} i d \tau\right\}=2 \int_{0}^{T} \operatorname{Re} \frac{\zeta^{\prime}(h+i \tau)}{\zeta(h+i \tau)} d \tau .
$$

Thus,

$$
2 \pi\left[N_{0}(T)+N_{1}(T)-1\right]=\operatorname{Im} \ln a\left(\frac{1}{2}-i T\right)+2 \operatorname{Im} \int_{h}^{1 / 2} \frac{\zeta^{\prime}(x+i T)}{\zeta(x+i T)} d x+2 \int_{0}^{T} \operatorname{Re} \frac{\zeta^{\prime}(h+i \tau)}{\zeta(h+i \tau)} d \tau .
$$

It yields

$$
\begin{aligned}
N_{0}(T) & +N_{1}(T)+\frac{1}{\pi} \operatorname{Im} \int_{1 / 2}^{h} \frac{\zeta^{\prime}(x+i T)}{\zeta(x+i T)} d x= \\
& =1+\frac{1}{2 \pi} \operatorname{Im} \ln a\left(\frac{1}{2}-i T\right)+\frac{1}{\pi} \int_{0}^{T} \operatorname{Re} \frac{\zeta^{\prime}(h+i \tau)}{\zeta(h+i \tau)} d \tau .
\end{aligned}
$$

Before a brief analysis of the obtained formula we denote by $\left\{T_{k}\right\}_{k=1}^{\infty}$ the monotonous and unboundedly increasing to $\infty$ sequence of $\zeta$-irregular values: $0<T_{1}<T_{2}<\cdots<T_{k} \uparrow+\infty$, and by $Q$ we denote an open set of all other $\left(\zeta\right.$-regular values) in $(0,+\infty): Q=\left(0, T_{1}\right) \cup\left(T_{1}, T_{2}\right) \cup \ldots$

We denote the right hand side of formula $(3)$ by $\mu_{1}(T)$. It is well-defined and continuous on $(0,+\infty)$ and, in particualr, at each $\zeta$-irregular point $T_{k}, k=1,2, \ldots$ The first two terms in formula (3) $N_{0}(T)+N_{1}(T)$ form a function (in notations of monograph [1] $N(T)=N_{0}(T)+$ $N_{1}(T)$, which is monotonous and unboundedly increasing on $(0,+\infty)$. At that, $N(T)$ is constant in each interval $\left(T_{k}, T_{k+1}\right)$ and at each point $T_{k}$ it has a jump of first kind equal to $\alpha_{k}+2 \beta_{k}$, where $\forall k \geqslant 1 \alpha_{k}$ is the multiplicity of possible zero of $\zeta(z)$ at the point $\frac{1}{2}+i T_{k}$, while $\beta_{k}$ is 
the sum of the multiplicities of all possible zeroes $\zeta(z)$ in the interval $\left(\frac{1}{2}+i T_{k}, h+i T_{k}\right)$; more precisely, in the segment $\left(\frac{1}{2}+i T_{k}, 1+i T_{k}\right]$. At that, we do not take into consideration and do not employ the well-known result by Vallée Poussin and Hadamard on the absence of zeroes of $\zeta(z)$ in the line $\operatorname{Re} z=1$. We always have $\alpha_{k}+2 \beta_{k} \geqslant 1, \forall k \geqslant 1$. In view of formula (3), function

$$
\mu_{2}(T):=\frac{1}{\pi} \operatorname{Im} \int_{1 / 2}^{h} \frac{\zeta^{\prime}(x+i T)}{\zeta(x+i T)} d x
$$

has a jump of first kind at each point $T_{k}$ and this jump is equal to $\alpha_{k}+2 \beta_{k}$. At that, $\forall k \geqslant 1$, $\forall T \in\left(T_{k}, T_{k+1}\right), \mu_{2}(T)=N(T)-\mu_{1}(T)=N\left(T_{k}\right)-\mu_{1}(T)$. Therefore, $\forall k \geqslant 1$ there exist finite one-sided limits

$$
\mu_{2}\left(T_{k}-0\right)=N\left(T_{k-1}\right)-\mu_{1}\left(T_{k}\right), \quad \mu_{2}\left(T_{k}+0\right)=N\left(T_{k}\right)-\mu_{1}\left(T_{k}\right) .
$$

Thus, function $\mu_{2}(T)$ is continuous in each interval in set $Q$ and in each $\zeta$-irregular point $T_{k}$ it has a jump of first kind equal to $N\left(T_{k}\right)-N\left(T_{k-1}\right)=\alpha_{k}+2 \beta_{k}$. We let $\psi(T)=\mu_{2}(T+0)-\mu_{2}(T-0)$. Then function $\psi(T)$ is defined in interval $(0,+\infty)$, vanishes at each point in $Q$ and is equal to $\alpha_{k}+2 \beta_{k}$ as $T=T_{k}, k \geqslant 1$.

Now we are in position to formulate one conjecture which seems to be plausible.

Conjecutre $\mathbf{A}_{\mathbf{0}}$. For each $T$ in $(0,+\infty)$ we have $|\psi(T)|<2$.

This conjecture implies

Theorem 2. Suppose that conjecture $A_{0}$ is true. Then

(1) Riemann conjecture on absence of zeroes of function $\zeta(z)$ in the half-plane $\operatorname{Re} z>1 / 2$ is true;

(2) all zeroes of $\zeta(z)$ in the line $\operatorname{Re} z=1 / 2$ are simple;

(3) the number $N_{0}(T)$ of all (simple) zeroes in the segment $\left[\frac{1}{2}, \frac{1}{2}+i T\right], T \in(0,+\infty)$, satisfies the relation

$$
N_{0}(T)=\frac{1}{2 \pi} \operatorname{Im} \ln a\left(\frac{1}{2}-i T\right)+\frac{1}{\pi} \operatorname{Im} \int_{1 / 2}^{h} \frac{\zeta^{\prime}(x+i T)}{\zeta(x+i T)} d x+O(1),
$$

where $h$ is an arbitrary fixed number in $(1,+\infty)$.

Statements (1) and (2) of this theorem are implied by the fact that the inequality $\alpha_{k}+2 \beta_{k}=$ $\left|\alpha_{k}+2 \beta_{k}\right|<2$ is possible for each $k \geqslant 1$ if and only if $\beta_{k}=0$ and $\alpha_{k}=1$. Then $\forall T \in(0,+\infty)$ (and for each fixed $h \in(1,+\infty)$ )

$$
\begin{aligned}
\left|\int_{0}^{T} \operatorname{Re} \frac{\zeta^{\prime}(h+i \tau)}{\zeta(h+i \tau)} d \tau\right| & =\left|\operatorname{Re} \int_{0}^{T} \frac{\zeta^{\prime}(h+i \tau)}{\zeta(h+i \tau)} d \tau\right|=|\ln | \zeta(h+i T)|-\ln | \zeta(h)|| \\
& \leqslant|\ln \zeta(h+i T)|+|\ln \zeta(h)| \leqslant M<+\infty .
\end{aligned}
$$

In order to employ formula (4), we need to establish asymptotic representation (as $T \gg 1$ ) for the function $\left|\operatorname{Im} \ln a\left(\frac{1}{2}-i T\right)\right|$ that can be done and to estimate the growth $($ as $T \uparrow \infty)$ of function

$$
\left|\operatorname{Im} \int_{1 / 2}^{h} \frac{\zeta^{\prime}(x+i T)}{\zeta(x+i T)} d x\right|
$$

that is a much more difficult problem. We recall that Bäcklund in his simpler proof (than the original one by von Mangoldt, see, for instance, [1]) proved that

$$
\left|\operatorname{Im} \int_{1 / 2}^{h} \frac{\zeta^{\prime}(x+i T)}{\zeta(x+i T)} d x\right|=O(\ln T) .
$$


It seems that this result by Bäcklund can be essentially specified.

We note that Conjecture $A_{0}$ (together with Theorem 2) will be disproved if at the line $\operatorname{Re} z=1 / 2$ there exists a multiple root (then there exist at least two such roots) of function $\zeta(z)$ or there exists at least one zero of $\zeta(z)$ (then there exist at least four such roots) belonging to the union of two vertical lines $0<\operatorname{Re} z<1 / 2,1 / 2<\operatorname{Re} z<1$.

We also observe that formulae (2)-(4) can be obtained by employing the theorem on logarithmic residue for other contours (like a rectangular tetragon and a right triangle).

\section{BIBLIOGRAPHY}

1. E.C. Titchmarsh. The theory of the Riemann zeta-function. Clarendon Press, Oxford (1951).

2. H.M. Edwards. Riemann's zeta function. Dover Publications, Inc., Mineola, New York (1974).

Yurii Fedorovich Korobeinik,

South Mathematical Institute, VSC RAS,

Markus str., 22,

362027, Vladikavkaz, Russia

South Federal University,

I.I. Vorovich Institute of mathematics, mechanics and computer sciences,

Milchakova str., 8a, 344090, Rostov-on-Don, Russia

E-mail: kor@math.rsu.ru 